\title{
Increased cytochrome $c$ correlates with poor survival in aggressive lymphoma
}

\author{
SARAH T. WILKINSON ${ }^{1}$, DAVID B.F. JOHNSON ${ }^{2}$, HEATHER L. TARDIF ${ }^{1}$, \\ MARGARET E. TOME ${ }^{1}$ and MARGARET M. BRIEHL ${ }^{1}$ \\ ${ }^{1}$ Department of Pathology, University of Arizona, Tucson, AZ 85724; ${ }^{2}$ Jack H. Skirball Center \\ for Chemical Biology and Proteomics, La Jolla, CA 92037, USA
}

Received July 23, 2009; Accepted October 1, 2009

DOI: 10.3892/ol_00000040

\begin{abstract}
Mitochondria are central to a variety of cellular processes, from metabolism to cell death. In this study, we demonstrated that an increase in the critical mitochondrial protein, cytochrome $c$, correlated with drug resistance in a cell culture model of aggressive lymphoma. Increased cytochrome $c$ expression was also correlated with decreased survival in the aggressive diffuse large B-cell and mantle cell lymphomas, but not in the indolent follicular lymphoma. This suggests that an increased reliance on the mitochondria for energy allows tumor cells to be metabolic opportunists and contribute to tumor development and drug resistance.
\end{abstract}

\section{Introduction}

Apoptosis resistance and altered metabolism are common cancer cell traits. In a murine lymphoma model, selection for oxidative stress resistance results in concomitant resistance to apoptosis and the acquisition of a more-aggressive tumor phenotype. WEHI7.2 thymic lymphoma cells transfected with catalase (CAT2, CAT38) or selected for resistance to hydrogen peroxide (200R) demonstrate resistance to glucocorticoid-induced apoptosis $(1,2)$, one component of the CHOP lymphoma chemotherapy regimen. The oxidative stress-resistant cells are also resistant to the cyclophosphamide, doxorubicin and vincristine components of CHOP (Tome et al, unpublished data). Catalase-overexpressing cells exhibit increased tumor growth compared to WEHI7.2 parental cells in a mouse xenograft model (2). Additionally, the oxidative stress-resistant cells demonstrate an altered metabolic profile, including the ability to generate ATP from alternative carbon sources such as glutamine $(3,4)$. When treated with glucocorticoids, the oxidative stress-resistant cells are better able to maintain ATP

Correspondence to: Dr Margaret M. Briehl, Department of Pathology, University of Arizona, 1501 N. Campbell Ave LSN 548, PO Box 245043, Tucson, AZ 85724, USA

E-mail:mmbriehl@email.arizona.edu

Key words: cytochrome $c$, gene expression profiling, diffuse large B-cell lymphoma, mantle-cell lymphoma, follicular lymphoma levels as compared to WEHI7.2 cells (3). Experiments with an uncoupler of mitochondrial respiration showed that the CAT38 and 200R cells produce more ATP from mitochondria than the WEHI7.2 cells (4).

Mitochondria are central to cellular metabolism and the decision to undergo apoptosis (5). Thus, changes in critical mitochondrial proteins may explain the more-aggressive tumor phenotype of the resistant lymphoma variants. In particular, cytochrome $c$ plays an integral role as an electron carrier in the mitochondrial respiratory chain, and the release of this protein from the mitochondrial intermembrane space results in commitment to death via apoptosis (5). In a previous study, we showed that cytochrome $c$ release is delayed in the resistant variants (1), and that mitochondria isolated from these cells demonstrate an intrinsic resistance to cytochrome $c$ release (Wilkinson et al, unpublished data). The present study examined whether alterations in cytochrome $c$ protein levels correlate with the previously characterized more-aggressive tumor phenotype of the resistant cells. To test the potential clinical relevance of these findings, gene expression data from lymphoma specimens were analyzed.

\section{Materials and methods}

Mitochondrial protein levels in cell lines. WEHI7.2 murine thymic lymphoma cells and variants were maintained as previously described (1,2). Mitochondria were isolated as previously described (2), with the exception that the mitochondrial isolation buffer was prepared in the same manner as Wang et al (6). SDS-PAGE and immunoblotting were performed using standard protocols. The antibodies used were: adenylate kinase-2 (AK-2) (Abcam, Cambridge, MA, USA); cytochrome $c$ (BD Biosciences, San Jose, CA, USA); HSP60 (Assay Designs, Ann Arbor, MI, USA); antirabbit-HRP (Cell Signaling Technology, Beverly, MA, USA) and anti-mouse-HRP (Pierce, Rockford, IL, USA).

Gene expression profiling data. The gene expression profiling (GEP) data sets used in this analysis were generated by the Leukemia/Lymphoma Molecular Profiling Project (LLMPP) research group (7-9) and are publicly available from http:// llmpp.nih.gov/. Diagnostic specimens were collected from 240 de novo diffuse large $\mathrm{B}$-cell lymphoma (DLBCL) 


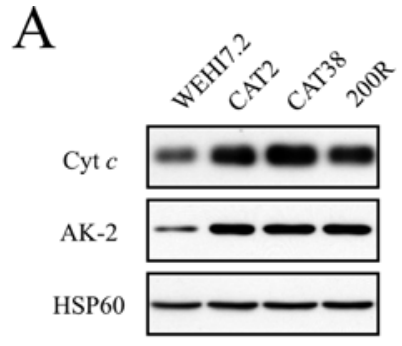

$\mathrm{C}$

Diffuse large B-cell lymphoma

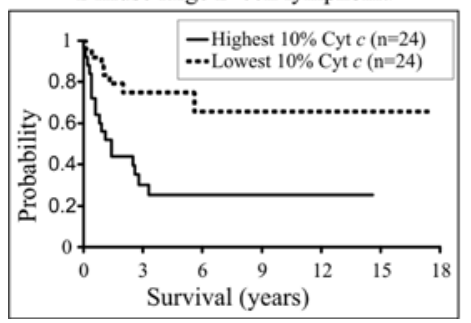

$\mathrm{B}$

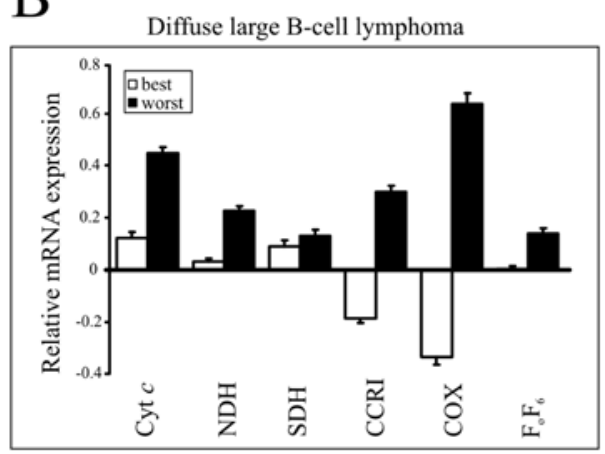

Figure 1. Increased expression of cytochrome $c$ in lymphoma. (A) Mitochondrial expression of cytochrome $c$, AK-2 and HSP60 proteins. Immunoblots of mitochondrial protein $(50 \mu \mathrm{g})$ from WEHI7.2 parental, CAT2, CAT38 and 200R cells were probed with antibodies to cytochrome $c$, AK-2 and HSP60. Representative immunoblots are shown. (B) Mean mRNA expression of oxidative phosphorylation proteins in diffuse large B-cell lymphoma patients with the worst prognosis vs. those with the best prognosis. Bars represent the mean relative mRNA expression on a log 2 scale, for the $10 \%$ of patients with the best (white bars) and worst (dark bars) outcome predictor score, plus or minus the standard error of the mean. (C) Overall survival of patients separated by cytochrome $c$ expression in diffuse large B-cell, mantle cell and follicular lymphoma. Kaplan-Meier plots were used to compare patient survival with the highest cytochrome $c$ expression (solid line) with that of patients with the lowest cytochrome $c$ expression (dashed line). The percentage of patients used for analysis is indicated on each graph. Cyt $c$, cytochrome $c$; NDH, NADH dehydrogenase; SDH, succinate dehydrogenase; CCRI, cytochrome $c$ reductase I; $\mathrm{COX}$, cytochrome $c$ oxidase; $\mathrm{F}_{\mathrm{O}} \mathrm{F}_{6}$, ATPase $\mathrm{F}_{\mathrm{O}} \mathrm{F}_{6}$.

(7), 92 mantle cell lymphoma (MCL) (9) and 191 follicular lymphoma (FL) patients (8). Patients subsequently received multi-agent chemotherapy and were monitored to assess treatment outcome. GEP was performed using the Lymphochip microarray for DLBCL and MCL and the Affymetrix U133A and U133B microarrays for FL.

Genes of interest were subjected to expressed sequence tag validation and statistical analysis as previously described (10). For cytochrome $c$ analyses, patients were categorized by increasing expression, separated into upper and lower expression groups and Kaplan-Meier plots were generated to compare survival between groups. To ensure that the number of patients studied would be similar among the lymphoma types, we compared the top and bottom $10 \%$ of patients in the larger DLBCL and FL data sets, as well as the top and bottom $20 \%$ of patients in the smaller MCL data set. Statistical tests were performed using WinSTAT for Microsoft Excel (Microsoft, Redmond, WA, USA).

\section{Results}

Increased mitochondrial cytochrome c protein expression in a drug-resistant lymphoma model. Cytochrome $c$ protein levels were increased in mitochondria from CAT2, CAT38 and 200R cells relative to WEHI7.2 (Fig. 1A). Increased protein levels were also noted for AK-2, another mitochondrial intermembrane space protein (Fig. 1A). This protein supports optimal ATP synthesis by equilibrating adenylate pools (11). The increased levels of cytochrome $c$ and AK-2 found in mitochondria from the oxidative stress-resistant lymphoma cells are proportionate to the degree of apoptotic resistance measured previously in intact cells (2). No difference in expression of the mitochondrial matrix protein HSP60 was found in the cell variants. Thus, the changes observed in cytochrome $c$ and AK-2 content cannot be explained by increased mitochondrial mass. These data suggest that the resistant variants have an increased mitochondrial respiratory capacity.

Correlation of increased oxidative phosphorylation (OXPHOS) proteins with poor prognosis in diffuse large $B$-cell lymphoma. Based on the characteristics of the lymphoma variants observed in this and a previous study (3), an increased expression of cytochrome $c$ and other genes involved in OXPHOS is expected to correlate with a poor response to chemotherapy in lymphoma patients. To test this, we analyzed a GEP data set from DLBCL patient samples. A previous study used this GEP data set to develop an outcome predictor score (OPS) that accurately predicted patient survival following chemotherapy (7). Ten genes coding for proteins involved in OXPHOS, including cytochrome $c$, were included on the LLMPP microarray (Table I). We first identified cytochrome $c$ as significantly correlated with OPS since an increased expression was associated with poor patient prognosis $(\mathrm{p}<0.05$; Table I and Fig. 1B). Additionally, the increased expression of NADH dehydrogenase, succinate dehydrogenase, cytochrome $c$ reductase $\mathrm{I}$, cytochrome $c$ 
Table I. Correlation of mitochondrial protein gene expression in diffuse large B-cell lymphoma patient tumor samples with prognosis ${ }^{\mathrm{a}}$.

\begin{tabular}{lcccc}
\hline & & & \multicolumn{2}{c}{ Statistical significance } \\
\cline { 2 - 5 } Gene & $\begin{array}{c}10 \% \text { best } \\
\text { prognosis } \\
\text { Mean }\end{array}$ & $\begin{array}{c}10 \% \text { worst } \\
\text { prognosis } \\
\text { Mean }\end{array}$ & $\begin{array}{c}\text { t-test; best 10\% } \\
\text { vs. Worst 10\% } \\
\text { P-value }\end{array}$ & $\begin{array}{c}\text { Regression } \\
\text { all patients } \\
\text { P-value }\end{array}$ \\
\hline Cytochrome $c$ & 0.1230 & 0.4475 & $0.0498^{\mathrm{c}}$ & $0.0001^{\mathrm{c}}$ \\
NADH dehydrogenase & 0.0315 & 0.2270 & $0.0486^{\mathrm{c}}$ & $0.0010^{\mathrm{c}}$ \\
Succinate dehydrogenase & 0.0899 & 0.1310 & 0.7941 & $0.0531^{\mathrm{c}}$ \\
Cytochrome $c$ reductase I & -0.1857 & 0.2987 & $0.0016^{\mathrm{c}}$ & $<0.0001^{\mathrm{c}}$ \\
Cytochrome $c$ reductase II & 0.2167 & 0.2987 & 0.5866 & 0.7159 \\
Cytochrome $c$ reductase bp & 0.0367 & 0.0739 & 0.7155 & 0.6744 \\
Cytochrome $c$ oxidase & -0.3338 & 0.6360 & 0.2291 & $<0.0001^{\mathrm{c}}$ \\
ATPase $\mathrm{F}_{\mathrm{O}} \mathrm{F}_{6}$ & 0.0022 & 0.1405 & 0.8237 & $0.0132^{\mathrm{c}}$ \\
ATPase $\mathrm{F}_{1} \beta$ & 0.1261 & 0.1604 & 0.9531 & 0.0872 \\
ATPase $\mathrm{F}_{\mathrm{O}} \mathrm{g}$ & 0.1425 & 0.1512 & 0.3684 \\
\hline
\end{tabular}

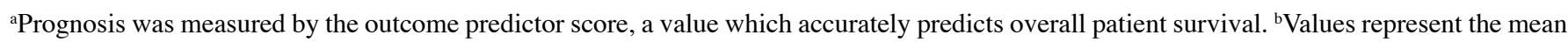
relative mRNA expression on a $\log 2$ scale for the $10 \%$ of patients with the best and worst prognosis from the LLMPP microarray. ${ }^{\mathrm{c} D e n o t e s}$ $\mathrm{p} \leq 0.05$.

oxidase and ATPase $\mathrm{F}_{\mathrm{O}} \mathrm{F}_{6}$ were significantly correlated with poor patient prognosis by OPS in DLBCL $(\mathrm{p}<0.05$; Table I and Fig. 1B). AK-2 expression was not significantly correlated (at $\mathrm{p} \leq 0.05$ ) with OPS. Notably, pyruvate dehydrogenase kinase 3 also showed significant correlation with OPS. Expression of this gene was significantly lower $(\mathrm{p} \leq 0.05)$ in the $10 \%$ of patients with the worst outcome. The kinase that it encodes functions to suppress the conversion of pyruvate to acetyl-CoA by pyruvate dehydrogenase (PDH). Other studies have shown that the fate of pyruvate can determine tumor metabolism pathways, with increased PDH stimulating mitochondrial respiration (12). Thus, the decreased expression of PDH kinase in DLBCL patients with the worst prognosis supports the increased activity of PDH, increased production of acetyl-CoA substrate for entry into the tricarboxylic acid cycle, and ultimately, the increased generation of mitochondrial ATP.

Correlation of increased cytochrome $c$ expression with poor survival in aggressive lymphoma. Given the results in our cell culture model and the correlation with survival following chemotherapy in DLBCL, we predicted that cytochrome $c$ levels would correlate with overall survival in lymphoma patients. We tested this using GEP data for three non-Hodgkin lymphomas: DLBCL, MCL and FL. In the aggressive DLBCL, survival was significantly worse $(\mathrm{p}<0.05)$ in the $10 \%$ of patients with the highest cytochrome $c$ expression compared to those with the lowest expression (Fig. 1C). Similarly, for MCL, another aggressive B-cell lymphoma, survival was significantly worse $(\mathrm{p}<0.05)$ in the $20 \%$ of patients with the highest cytochrome $c$ expression compared to those with the lowest expression (Fig. 1C). For FL, an indolent B-cell lymphoma, the outcome was different. Cytochrome $c$ expression did not correlate with overall survival in FL (Fig. 1C).

\section{Discussion}

This study places mitochondria in the crossroads of successful adaptation to oxidative stress and carcinogenesis. The mitochondrial changes observed in our lymphoma model extend previous findings of metabolic flexibility in the oxidative stress-resistant cells $(3,4)$. Our attempts to use continuous ethidium bromide treatment as a technique to deplete mitochondrial DNA (13) with the WEHI7.2 cells were unsuccessful (data not shown). The inability to create WEHI7.2 rho $^{0}$ cells lacking functional OXPHOS suggests that reliance on mitochondrial respiration is critical for lymphoma cells. This complements the observed increase in cytochrome $c$ and AK-2 levels, as well as an increased ability to maintain mitochondrial ATP generation (3), in the resistant variants. A novel conclusion from the present study, building on our previous biochemical analyses $(3,4)$, is that oxidative stress may provide selective pressure for the concurrent development of apoptosis resistance and the metabolic adaptations seen in malignant cells. Since lymphoma can arise in the context of chronic infection and inflammation, our investigations may have implications for both current and novel therapies.

Increasing the mitochondrial respiratory proteins allows the oxidative stress resistant variants to be metabolic opportunists. Studies in HeLa cells show that growth in galactose/ glutamine instead of glucose leads to increased dependence on OXPHOS (14). This biochemical change occurs via increased respiratory chain protein levels without increasing the mitochondrial mass. Thus, an increase in respiratory chain proteins, such as cytochrome $c$, may indicate enhanced OXPHOS capability and account for metabolic flexibility in the aggressive variants.

Our analyses show an increased expression of cytochrome $c$ and other mitochondrial respiration proteins in aggressive 
lymphomas from patients with the worst prognosis, suggesting that results from the lymphoma cell model are clinically relevant. The up-regulation of respiratory chain proteins in the DLBCL patients with the worst prognosis is consistent with an ability to use alternative energy substrates such as glutamine. Some studies suggest that tumor cells suppress OXPHOS as a protective mechanism to limit the production of endogenous reactive oxygen species (12). However, in our oxidative stressresistant model and in tumors arising in the context of chronic inflammation, the ability to tolerate increased oxidative stress may allow them to take advantage of increased mitochondrial respiration.

We have shown that an increased cytochrome $c$ expression is correlated with decreased survival in the aggressive DLBCL and MCL. The up-regulation of alternate pathways for ATP production would allow cancer cells to be metabolic opportunists in a tumor microenvironment where specific nutrients are limiting. This could provide both a growth advantage during tumor development, as well as protection from apoptosisinducing chemotherapy. A correlation between cytochrome $c$ expression and overall survival was not observed in the indolent FL, suggesting that for a less-aggressive cancer, increased OXPHOS capability would be less critical. Additionally, the majority of follicular lymphoma cases are characterized by overexpression of $B C L 2$ (8), and it is possible that this prominent survival advantage overshadows metabolic changes.

Our results suggest that an increased ability to use mitochondria to produce ATP contributes to tumor development. The WEHI7.2 variants with the mitochondrial alterations are resistant to multiple lymphoma chemotherapeutics (Tome et al, unpublished data), suggesting that these mitochondrial characteristics also contribute to chemoresistance in the clinic. Targeting such mitochondrial alterations with novel chemotherapeutics may improve clinical outcome for these aggressive lymphomas.

\section{Acknowledgements}

Funding was from NCI CA71768 (M.M.B), NCI-T32 CA09213 (S.T.W. and H.L.T.), HHMI 52003749 (D.B.F.J.), and ARCS Foundation, Phoenix Chapter (H.L.T.).

\section{References}

1. Tome ME and Briehl MM: Thymocytes selected for resistance to hydrogen peroxide show altered antioxidant enzyme profiles and resistance to dexamethasone-induced apoptosis. Cell Death Differ 8: 953-961, 2001.

2. Tome ME, Baker AF, Powis G, Payne CM and Briehl MM: Catalase-overexpressing thymocytes are resistant to glucocorticoid-induced apoptosis and exhibit increased net tumor growth. Cancer Res 61: 2766-2773, 2001.

3. Tome ME, Lutz NW and Briehl MM: Overexpression of catalase or Bcl-2 alters glucose and energy metabolism concomitant with dexamethasone resistance. Biochim Biophys Acta 1693: 57-72, 2004.

4. Tome ME, Briehl MM and Lutz NW: Increasing the antioxidant defense in WEHI7.2 cells results in a more tumor-like metabolic profile. Int J Mol Med 15: 497-501, 2005.

5. Newmeyer DD and Ferguson-Miller S: Mitochondria: releasing power for life and unleashing the machineries of death. Cell 112: 481-490, 2003.

6. Wang GQ, Gastman BR, Wieckowski E, Goldstein LA, Rabinovitz A, Yin XM and Rabinowich H: Apoptosis-resistant mitochondria in $\mathrm{T}$ cells selected for resistance to Fas signaling. $\mathrm{J}$ Biol Chem 276: 3610-3619, 2001

7. Rosenwald A, Wright G, Chan WC, et al: The use of molecular profiling to predict survival after chemotherapy for diffuse largeB-cell lymphoma. N Engl J Med 346: 1937-1947, 2002.

8. Dave SS, Wright G, Tan B, et al: Prediction of survival in follicular lymphoma based on molecular features of tumor-infiltrating immune cells. N Engl J Med 351: 2159-2169, 2004.

9. Rosenwald A, Wright G, Wiestner A, et al: The proliferation gene expression signature is a quantitative integrator of oncogenic events that predicts survival in mantle cell lymphoma. Cancer Cell 3: 185-197, 2003.

10. Tome ME, Johnson DB, Rimsza LM, Roberts RA, Grogan TM, Miller TP, Oberley LW and Briehl MM: A redox signature score identifies diffuse large B-cell lymphoma patients with a poor prognosis. Blood 106: 3594-3601, 2005.

11. Igamberdiev AU and Kleczkowski LA: Equilibration of adenylates in the mitochondrial intermembrane space maintains respiration and regulates cytosolic metabolism. J Exp Bot 57: 2133-2141, 2006.

12. Frezza $\mathrm{C}$ and Gottlieb E: Mitochondria in cancer: not just innocent bystanders. Semin Cancer Biol 19: 4-11, 2009.

13. King MP and Attardi G: Isolation of human cell lines lacking mitochondrial DNA. Methods Enzymol 264: 304-313, 1996.

14. Rossignol R, Gilkerson R, Aggeler R, Yamagata K, Remington SJ and Capaldi RA: Energy substrate modulates mitochondrial structure and oxidative capacity in cancer cells. Cancer Res 64: 985-993, 2004. 